\title{
Shape Sensing of Plate and Shell Structures Undergoing Large Displacements Using the Inverse Finite Element Method
}

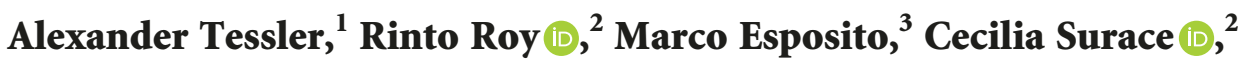 \\ and Marco Gherlone $\mathbb{1}^{3}$ \\ ${ }^{1}$ NASA Langley Research Center, Structural Mechanics and Concepts Branch, Hampton, VA, USA \\ ${ }^{2}$ Politecnico di Torino, Department of Structural, Geotechnical and Building Engineering, Torino, Italy \\ ${ }^{3}$ Politecnico di Torino, Department of Mechanical and Aerospace Engineering, Torino, Italy \\ Correspondence should be addressed to Cecilia Surace; cecilia.surace@polito.it
}

Received 25 May 2018; Revised 9 September 2018; Accepted 16 September 2018; Published 25 October 2018

Academic Editor: Giosuè Boscato

Copyright (c) 2018 Alexander Tessler et al. This is an open access article distributed under the Creative Commons Attribution License, which permits unrestricted use, distribution, and reproduction in any medium, provided the original work is properly cited.

\begin{abstract}
The inverse Finite Element Method (iFEM) is applied to reconstruct the displacement field of a shell structure which undergoes large deformations using discreet strain measurements as the prescribed data. The iFEM computations are carried out using an incremental procedure where at each load step, the incremental strains are used to evaluate the incremental displacements which in turn update the geometry of the deformed structure. The efficacy of the proposed approach to predict large displacements is examined using two case studies involving a cantilevered wing-shaped plate and a clamped plate. The incremental iFEM procedure is demonstrated to be sufficiently accurate in terms of reproducing the correct nonlinear character of the loaddisplacement curve even when a reduced number of strain sensors is used. Therefore, this approach may have important implications for real-time monitoring of aerospace structures that undergo large displacements.
\end{abstract}

\section{Introduction}

Efficient structural health monitoring (SHM) systems are enabling technologies for the maintenance and control of future aerospace vehicles. When implemented, such systems provide real-time monitoring of the vehicle's structural integrity. This technology would thus enable improved structural safety, reduced maintenance operations, and significant cost savings. A SHM system uses a set of strain sensors embedded on the surface of the structure. Using real-time sensor measurements, the structural deformations need to be reconstructed, thus requiring the solution of an inverse problem known as "shape sensing".

Existing shape-sensing methods can be classified into four categories based on different approaches [1]: (1) numerical integration of experimental strains, (2) use of global or piecewise continuous basis functions to approximate the displacement field, (3) application of neural networks, and (4) use of variational approaches. Most of the methods based on the integration of measured strains deal with beam problems and make use of the classical beam equations $[2,3]$. For the full-field estimation of the deformed shape of more complex two-dimensional structures, basis-function approaches utilize an a priori set of spatial functions and unknown coefficients to fit discretely measured strains [4-8]. A major drawback of the methods based on neural networks is that their accuracy depends on the choice of the training load cases [9].

For many large-scale practical applications, the variational methods appear to be most attractive. In these methods, a suitable error functional is minimized over the entire problem domain, by comparing the estimated and measured strains, commonly in the least-squares sense. One such method is the inverse Finite Element Method (iFEM), advanced by Tessler and Spangler [10-12] and initially developed for shear-deformable plate and shell structures. The method employs a weighted least-square variational formulation by discretizing the problem domain using $\mathrm{C}^{0}$ continuous finite elements. The approach is suitable for 
modelling complex geometries and allows both static and dynamic responses to be reconstructed without any prior knowledge of material properties or the loading conditions of the structure. The inverse Finite Element Method was adapted by Gherlone [13] for shape sensing of truss, beam, and frame structures.

Since the initial introduction of iFEM, the approach has been successfully applied on a variety of simple and complex structures, where the focus has been primarily on the small deformation, linear load-displacement response. The existing iFEM formulations use the simplifying assumption of "small displacements"; hence, applying these methods to large-displacement problems will generally result in significant errors in the prediction of the deformed shape.

The first real attempt at addressing the problem of geometric nonlinearity was made by Tessler et al. [14]. The method involves a modification of the standard linear iFEM, where the linear iFEM is used over a number of load steps and the incremental variation in deformation over each step is summed to determine the final deformed shape of the structure. This method bears resemblance to the nonlinear finite element method, which also uses a series of linear displacement increments to recreate the nonlinear nature of the load-deflection curve. Initial results obtained by employing this technique to predict deformations in a complex wing profile were seen as promising.

Shape-sensing techniques capable of predicting large deformations have a wide range of applications for future aircraft and spacecraft missions. Many aircraft components, such as the fuselage skin and certain parts of the wing, are subjected to compressive loading during aircraft operation. An aircraft structural health monitoring system capable of reconstructing the nonlinear structural response should facilitate improved predictions of the onset of buckling and related failure. Such technologies can also be applied to shape morphing structures in aircraft and membrane space structures such as solar sails, spacecraft deorbiters, and sun shields (as in the James Webb Space Telescope), where large deformations are normally observed. The predicted shape can be used as feedback for the actuation system to facilitate control over the deformed structural shape.

This paper aims to describe the basic methodology and procedure of the linear incremental iFEM formulation and to demonstrate the method's suitability to reconstruct large deformations in plate and shell structures. In Section 2, the formulation of the standard linear iFEM is briefly described, followed by the linear incremental iFEM procedure. In Section 3, the iFEM approach for predicting large deformations is demonstrated on two example problems, one involving a cantilevered wing-shaped plate and the other, a clamped square plate under transverse loading. The effect of the number of strain sensors on the accuracy of the reconstructed displacement field is also investigated. Finally, concluding remarks and future directions of this research are presented in Section 4.

\section{The Inverse Finite Element Method}

In this section, a brief review of the iFEM approach for applications to beam, plate, and shell structures is presented.
Let us consider the structural domain $\Omega$ to be discretized using one-, two-, or three dimensional finite elements [11-13]. The displacement field experienced by the structure is completely defined by the vector $\left(u_{x}(\mathbf{x}), u_{y}(\mathbf{x}), u_{z}(\mathbf{x})\right)$ in the Cartesian coordinate system $\mathbf{x} \equiv(x, y, z)$. Depending on the particular structural theory, the displacement vector can be expressed in terms of a set of kinematic variables $\mathbf{u}(\mathbf{x})$. These kinematic variables can be interpolated within each finite element of the discretization by a set of element-shape functions:

$$
\mathbf{u}(\mathbf{x}) \approx \mathbf{u}^{e}=\mathbf{N}(\mathbf{x}) \mathbf{q}^{e},
$$

where $\mathbf{N}$ is the shape-function matrix and $\mathbf{q}^{e}$ the nodal degrees-of-freedom.

Corresponding to the adopted structural theory, the strain field is completely described by a set of $K$ independent quantities, $\varepsilon \equiv\left\{\varepsilon_{k}\right\}(k=1, \ldots, K)$, usually referred to as strain measures. Using Equation (1) and the linear straindisplacement relations, the strain measures can be expressed in terms of the nodal degrees-of-freedom:

$$
\boldsymbol{\varepsilon}\left(\mathbf{u}^{e}\right)=\mathbf{L}(\mathbf{x}) \mathbf{q}^{e},
$$

where the matrix $\mathbf{L}$ contains the derivatives of the shape functions.

The iFEM approach is based on the minimization of a functional defined as the least-squares error between the analytic strain measures (Equation (2)) and the corresponding experimental strain measures, $\mathcal{\varepsilon}^{\varepsilon} \equiv\left\{\varepsilon_{k}^{\varepsilon}\right\}(k=1, \ldots, K)$. The latter are evaluated at $n$ discrete locations using strain gauges, strain rosettes, or other types of strain sensors. For a single element, the error functional, $\Phi^{e}$, is defined as the sum of the products of the least-squares component, $\Phi_{k}^{e}$, referred to the $k$-th strain measure, and the corresponding weighting coefficient, $w_{k}^{e}$,

$$
\Phi^{e}\left(\mathbf{u}^{e}\right) \equiv \sum_{k} w_{k}^{e} \Phi_{k}^{e}
$$

with $\Phi_{k}^{e}$ defined as

$$
\Phi_{k}^{e} \equiv \frac{1}{n} \sum_{i=1}^{n}\left[\varepsilon_{k(i)}\left(\mathbf{u}^{e}\right)-\varepsilon_{k(i)}^{\varepsilon}\right]^{2}, \quad(k=1, \ldots, K) .
$$

The weighting coefficients $w_{k}^{e}$ are the products of dimensional parameters and dimensionless coefficients, $\lambda_{k}^{e}$. The dimensional parameters guarantee that the terms in Equation (3) have the same mathematical units [11-13], whereas the $\lambda_{k}^{e}$ coefficients are assigned either high (1, unity) or relatively low values $\left(10^{-4}\right)$ to enforce a stronger or weaker correlation between the analytic strain measures and their experimentally measured counterparts.

The inverse-element error functional, $\Phi^{e}$, is minimized with respect to the unknown nodal degrees-of-freedom, $\mathbf{q}^{e}$, leading to the element matrix equation $\mathbf{a}^{e} \mathbf{q}^{e}=\mathbf{b}^{e}$. Applying the appropriate coordinate transformations, the element contributions are assembled into a global system of equations, and upon enforcing problem-dependent displacement boundary conditions, the global equations in matrix form are

$$
\mathbf{A q}=\mathbf{b} .
$$


In Equation (5), the matrix A depends on the shape functions and strain-sensor locations, whereas the vector $\mathbf{b}$ is a function of the experimental strain measures. Equation (5) can be readily solved for the unknown nodal degrees-offreedom, $\mathbf{q}$ by inverting the matrix $\mathbf{A}$. Thus, for small displacements, the strain-sensor locations are assumed to remain unchanged, and $\mathbf{A}$ is inverted only once, whereas the vector $\mathbf{b}$ is updated at each strain-data acquisition increment.

Since only strain-displacement relations are used in the definition of $\Phi^{e}$, iFEM does not require the knowledge of any material properties or the applied loading. Thus, it is applicable for both static and dynamic conditions, without requiring inertial or damping material properties.

2.1. Plate Formulation. Consider a plate of thickness $2 t$ for which the $(x, y)$ plane of the Cartesian coordinate system represents the midplane (Figure 1). The components of the displacement vector are given according to Mindlin plate theory, as

$$
\begin{aligned}
& u_{x}(x, y, z)=u(x, y)+z \theta_{y}(x, y), \\
& u_{y}(x, y, z)=v(x, y)+z \theta_{x}(x, y), \\
& u_{z}(x, y, z) \approx w(x, y),
\end{aligned}
$$

where the five kinematic variables are $\mathbf{u} \equiv\left\{u, v, w, \theta_{x}, \theta_{y}\right\}^{T}$; $u$ and $v$ are average uniform displacements in the $x$ and $y$ directions, respectively; $w$ is the average transverse deflection; $\theta_{x}$ and $\theta_{y}$ are rotations of the normal about the negative $x$ and positive $y$ axes, respectively.

The strain field is identified by eight strain measures, three membrane $(k=1-3)$, three bending $(k=4-6)$, and two transverse shear $(k=7,8),\left\{\varepsilon_{k}\right\}(k=8)$, given by

$$
\begin{aligned}
& \mathbf{e}=\left\{u_{x}, v_{y}, \theta_{x, y}+\theta_{y, x}\right\}^{T}=\left\{\varepsilon_{1}, \varepsilon_{2}, \varepsilon_{3}\right\}^{T}, \\
& \mathbf{k}=\left\{\theta_{y, x}, \theta_{x, y}, \theta_{x, x}+\theta_{y, y}\right\}^{T}=\left\{\varepsilon_{4}, \varepsilon_{5}, \varepsilon_{6}\right\}^{T}, \\
& \mathbf{g}=\left\{w_{x}+\theta_{y}, w,_{y}+\theta_{x}\right\}^{T}=\left\{\varepsilon_{7}, \varepsilon_{8}\right\}^{T} .
\end{aligned}
$$

If Reference [12], a three-node inverse plate/shell element, iMIN3, has been developed and will be applied in the present analysis. The element is formulated using $C^{0}$ continuous anisoparametric shape functions to avoid shear locking. The deflection variable is interpolated with a quadratic polynomial, whereas the other four kinematic variables vary linearly over the element. The strain measures defined in Equation (7) can then be expressed in terms of the nodal degrees-of-freedom, as in Equation (2), and the error functional can be written as in Equations (3) and (4). The weighting coefficients are $\left\{w_{k}^{e}\right\}=\left\{\lambda_{1}^{e}, \lambda_{2}^{e}, \lambda_{3}^{e}, t^{2} \lambda_{4}^{e}, t^{2} \lambda_{5}^{e}, t^{2} \lambda_{6}^{e}, \lambda_{7}^{e}, \lambda_{8}^{e}\right\}$, where $\lambda_{k}^{e}(k=$ $1, \ldots, 8)$ are dimensionless, positive constants.

The membrane and bending curvature strain measures, $\mathbf{e}_{(i)}^{\varepsilon}$ and $\mathbf{k}_{(i)}^{\varepsilon}$, can be evaluated at the location $\left(x_{(i)}, y_{(i)}\right)$ from surface strain measurements on the top $(+)$ and bottom $(-)$ surfaces (Figure 2) as

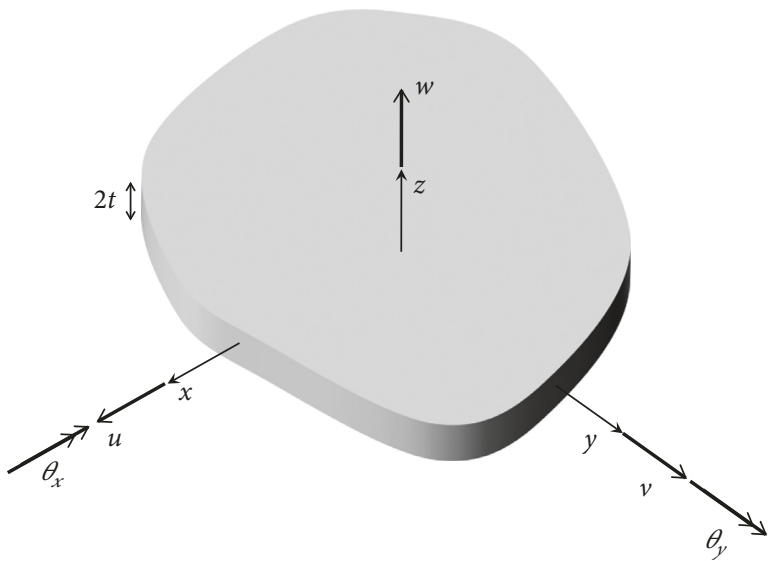

Figure 1: Plate geometry and kinematic variables.

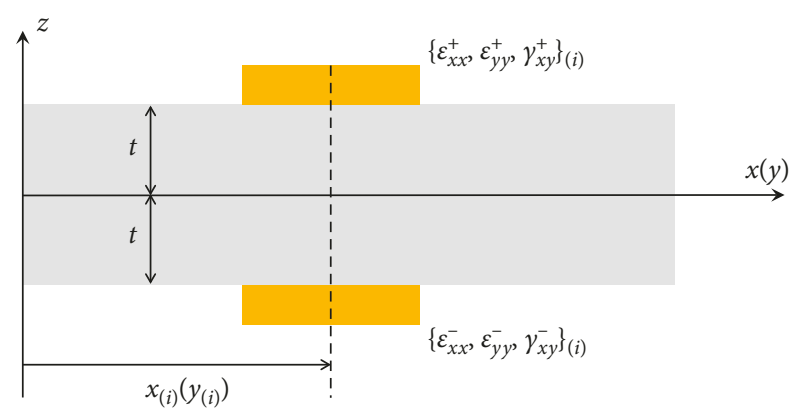

Figure 2: Strains measurement.

$$
\begin{aligned}
& \mathbf{e}_{(i)}^{\varepsilon}=\frac{1}{2}\left\{\left(\varepsilon_{x x}^{+}+\varepsilon_{x x}^{-}\right),\left(\varepsilon_{y y}^{+}+\varepsilon_{y y}^{-}\right),\left(\gamma_{x y}^{+}+\gamma_{x y}^{-}\right)\right\}_{(i)}^{T}, \\
& \mathbf{k}_{(i)}^{\varepsilon}=\frac{1}{2 t}\left\{\left(\varepsilon_{x x}^{+}-\varepsilon_{x x}^{-}\right),\left(\varepsilon_{y y}^{+}-\varepsilon_{y y}^{-}\right),\left(\gamma_{x y}^{+}-\gamma_{x y}^{-}\right)\right\}_{(i)}^{T} .
\end{aligned}
$$

The transverse shear strain measures, g, cannot be evaluated experimentally. Thus, for the corresponding terms in the functional (Equation (3)), the following expression is adopted $(k=7,8)$ :

$$
\Phi_{k}^{e} \equiv \int_{A^{e}} \varepsilon_{k(i)}^{2}\left(\mathbf{u}^{e}\right) d A,
$$

where $A^{e}$ is the element area. Moreover, $\lambda_{k}^{e}(k=7,8)$ are set to small values, e.g., $10^{-4}$, relative to 1 (unity) which is used for the $\lambda_{k}^{e}(k=1, \ldots, 6)$ coefficients.

When only few strain sensors are available, some elements may not have any strain data. For the elements without the strain data, the norms in Equation (9) are used for all eight strain measures, where the $\lambda_{k}^{e}(k=1, \ldots, 8)$ coefficients are assigned small values, e.g., $10^{-4}$, relative to 1 (unity) which is used for the elements that possess strain data.

\section{2. iFEM Incremental Algorithm for Nonlinear Deformations.} Herein, it is assumed that the structure under consideration is instrumented with a high-speed strain-sensor measurement system which is capable of measuring strain levels in 
real time as the structure undergoes deformations under quasistatic or dynamic loading. The standard linear iFEM formulation can be applied at each incremental load level to reconstruct the current deformed shape, using the current strain increments, and then updating the geometry of the iFEM mesh and repeating the shape-sensing analysis based on the next strain increment. Thus, geometrically nonlinear deformations ("large displacements") can be reconstructed in real time as the strain history becomes available to iFEM from a strain-sensor measurement system [13].

(i) At each load increment, $i$, evaluate (experimentally or with a nonlinear direct FEM analysis) the incremental section strains that represent measured strain increments.

(ii) Perform iFEM analysis using the strain increments to obtain the nodal-degrees-of-freedom, $\mathbf{q}_{i}$.

(iii) Update the structural geometry (i.e., the iFEM nodal coordinate positions) using the iFEM determined displacements, i.e., $\mathbf{x}_{i+1}=\mathbf{x}_{i}+\mathbf{N}\left(\mathbf{x}_{i}\right) \mathbf{q}_{i}$

(iv) Update the orientation of the measured strains corresponding to the current geometry, $\mathbf{x}_{i+1}$

(v) Repeat the above-stated incremental procedure until the strain history is complete.

The iFEM incremental procedure just outlined is thus in complete agreement with the standard incremental procedures used for geometrically nonlinear analysis of the direct FEM. Unlike the direct FEM, the iFEM incremental procedure does not invoke equilibrium iterations at different load increments. This is because iFEM has no access to equilibrium equations. Instead, the iFEM equations smooth the measured strain data and integrate the straindisplacement relations. Since the in-situ measured strains are constantly updated at each load level, the straindisplacement relations are constantly updated and corrected to the current state of deformation. Thus, within this incremental formulation, the strain-level updates provide the necessary corrections at each load increment. Consequently, as will be demonstrated in Section 3, highly accurate geometrically nonlinear deformations can be reconstructed by this simple and computationally efficient incremental iFEM procedure.

\section{Numerical Examples}

The application of the incremental iFEM method is presented using two example problems for elastostatic deformation of plates subjected to transverse loading. Nonlinear direct FE models are used both to provide the strain measurements as input data and displacements as reference results for the iFEM analysis. The FEM analyses are carried out using the finite-strain three-node S3R shell element in ABAQUS/Standard 6.13 [15]. The iFEM reconstruction is performed using the same triangular mesh, where the strain sensors are positioned on both the top and bottom surfaces of the iMIN3 elements.

The accuracy of the iFEM prediction is then assessed by calculating the Root Mean Square (RMS) of the nodal transverse deflection error with respect to the direct FEM results, defined as

$$
\% e_{\mathrm{RMS}}=100 \sqrt{\frac{1}{m} \sum_{i=1}^{n}\left(\frac{w_{i}^{\mathrm{iFEM}}-w_{i}^{\mathrm{FEM}}}{w_{\max }^{\mathrm{FEM}}}\right)^{2}},
$$

where $m$ indicates the total number of nodes, which is the same for both the direct and the inverse analyses.

In the first example [1], a wing-shaped cantilevered plate is considered (Figure 3(a)). The material is an aluminum alloy (Young's modulus $E=72017 \mathrm{MPa}$, Poisson's ratio $v=0.325$ ). The plate is subjected to a uniformly distributed transverse pressure providing a resultant load that corresponds to 30 times the plate's own weight. Figure $3(\mathrm{~b})$ shows the direct and inverse triangular mesh. Single-strain component measurements, simulating linear strain gauges, are provided to a subset of inverse elements of the iFEM discretization. In particular, 44 elements have linear strain gauges positioned on the top and bottom surfaces (Figure 3(b)). For all the inverse elements, $\lambda_{k}^{e}(k=7,8)=10-{ }^{4}$ has been used for the contributions to the error functional due to shear strain measures, whereas $\lambda_{k}^{e}=10^{-4}$ has been used for all of the contributions related to "non-measured" strain components or to elements without strain data.

Figure 4 shows the comparison between the loaddeflection curves as obtained using the nonlinear direct FEM and iFEM analyses. The maximum deflection experienced at the end of the loading process is comparable to the wing span, and the direct solution exhibits significant nonlinearity in the load-deflection curve. By using the standard linear iFEM procedure based on the final value of the "measured" strains, the reconstructed load-deflection response cannot reproduce the correct structural response. When adopting the incremental iFEM procedure, the loaddeflection curve is more accurate (Figure 5 shows that the RMS percent error never exceeds 1\%).

Figure 6 compares the deformed shapes of the plate obtained using the nonlinear direct FEM analysis and the linear incremental iFEM reconstruction.

In the second example, an isotropic square plate which is clamped along the four edges is subjected to a static pressure load of magnitude $5 \mathrm{MPa}$ on the top surface. The plate's side span is $5 \mathrm{~m}$ and the thickness is $10 \mathrm{~mm}$. The elastic modulus and Poisson's ratio are, respectively, $E=200000 \mathrm{MPa}$ and $v=$ 0.2 .

The iFEM analysis used the same triangular mesh as the direct FEM analysis, as depicted in Figure 7, and was supplied with a complete set of strain values at the element centroids.

Figure 8 shows the load-deflection curve for the transverse deflection at the center of the square plate. The linear iFEM was employed incrementally across a total of 21 load steps. The resulting highly nonlinear load-deflection curve is primarily due to stress stiffening. Even though the straindisplacement relations are kept linear at any load step, the gradual updating of the deformed geometry over a number of steps allows the incremental iFEM procedure to reproduce the highly accurate nonlinear deflections. In 


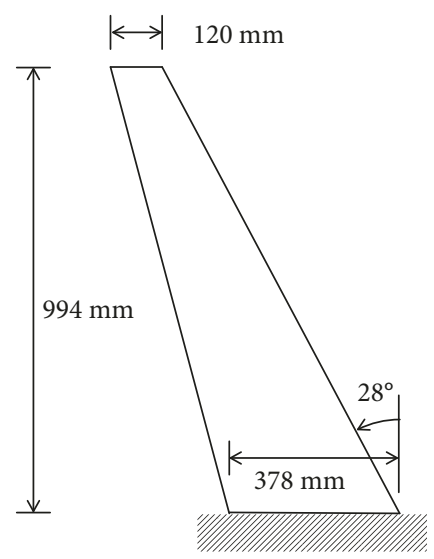

(a)

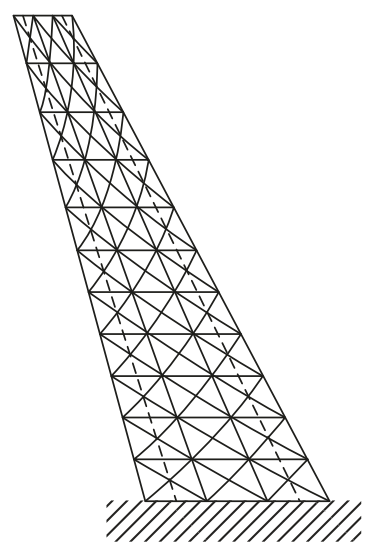

(b)

FIGURE 3: Wing-shaped plate model. (a) Geometry and boundary conditions (thickness $3 \mathrm{~mm}$ ) and (b) triangular mesh for both FEM and iFEM analysis (132 elements), 44 inverse elements with linear strain gauges oriented along two sensing lines (for a total of 88 strain measurements).

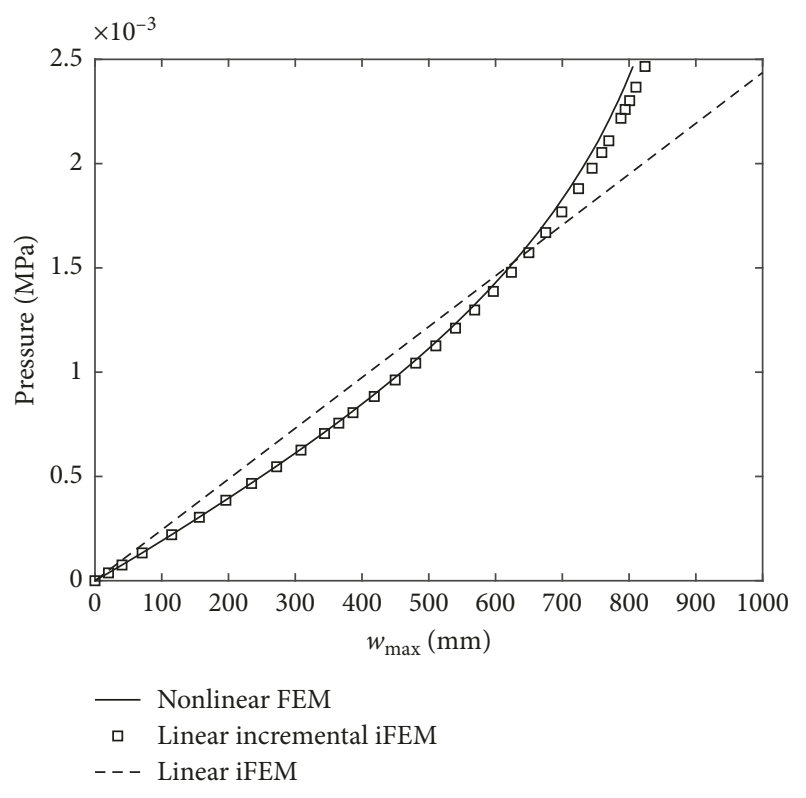

Figure 4: Wing-shaped plate. Load-deflection curves corresponding to the nonlinear direct FEM and linear iFEM solutions. The maximum deflection $\left(w_{\max }\right)$ is located at the intersection between the tip chord and the trailing edge.

comparison, the standard single-step linear iFEM application is represented by a straight line from the initial to the final deflection point, completely missing any intermediate nonlinear response. Note that the load-deflection curve of the nonlinear direct FEM analysis matches closely with the incremental iFEM results. The results are also examined further by computing the RMS error at each load step.

Figure 9 depicts the RMS error of the maximum deflection for each load step. Throughout the loading cycle, the linear incremental iFEM procedure results in the RMS error of less than $2 \%$. In contrast, the linear iFEM procedure results in substantially higher RMS error, reaching values of around $40 \%$ during the loading process.

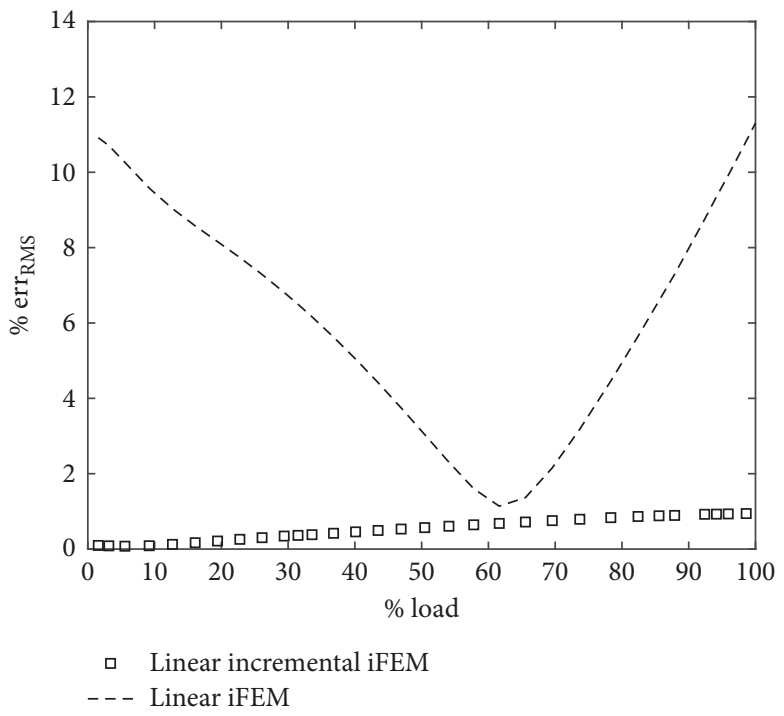

FIGURE 5: Wing-shaped plate. RMS percent error corresponding to linear iFEM and linear incremental iFEM solutions as a function of the load range.

Figure 10 shows the final deformed shape obtained by ABAQUS and the one predicted by the iterative iFEM procedure.

To ascertain the effect of the number of strain sensors and their locations on the predictive capability of the linear incremental iFEM procedure, a parametric study is performed for the clamped square plate problem. Six different strain-sensor configurations are selected (refer to Figure 11) to perform the parametric study. Each strain sensor is indicated by a red circle and shows the elements in which the strains are "measured" at the element centroids, on both top and bottom surfaces. The elements without the strain sensors are formulated according to Equation (9), using $\lambda_{k}^{e}=10^{-4}(k=1, \ldots, 8)$.

The total number of strain sensors used in each configuration is different, but care is taken to ensure that each 


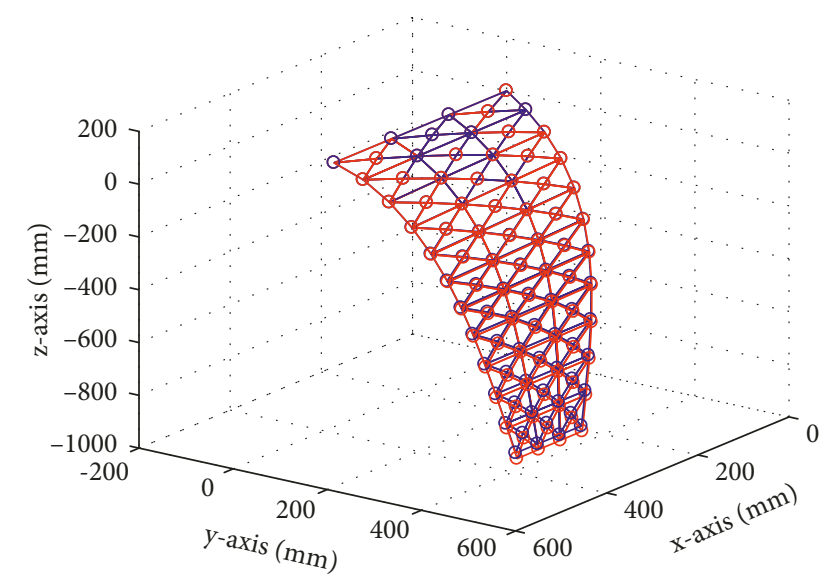

FIgURE 6: Wing-shaped plate. Deformed shape at the maximum load corresponding to the nonlinear direct FEM (blue) and linear incremental iFEM (red) solutions.

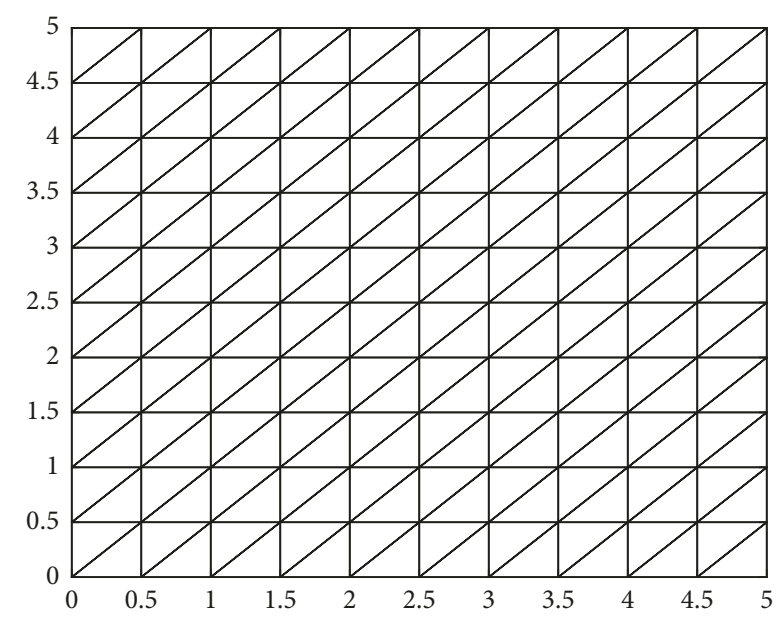

Figure 7: Clamped square plate. Triangular discretization of 200 elements for both the direct and inverse analyses.

configuration has a uniform distribution of strain sensors throughout the surface area of the plate to avoid any errors due to highly localised placement of sensors. Each configuration is characterised using the parameter $\beta$, which is defined as the average number of strain sensors in a $1 \mathrm{~m}^{2}$ area of the plate. The parameter $\beta$ is proposed with the intention of finding the minimum sensor density required for accurate shape sensing of nonlinear deflection of the considered plate structure.

The results from the linear incremental iFEM analysis are shown in Figure 12. As expected, higher values of $\beta$ result in lower RMS errors. As the value of $\beta$ increases, the RMS errors reduces and is seen to saturate starting at $\beta=4$. For $\beta$ values as low as 2 , the error magnitude is seen to be around $2 \%$, which is not a significant variation from the minimum error value of $1.61 \%$. These results indicate that even when using only 50 strain sensors, i.e., one quarter of the number of elements, the linear incremental iFEM procedure is capable of reproducing the nonlinear displacement field with

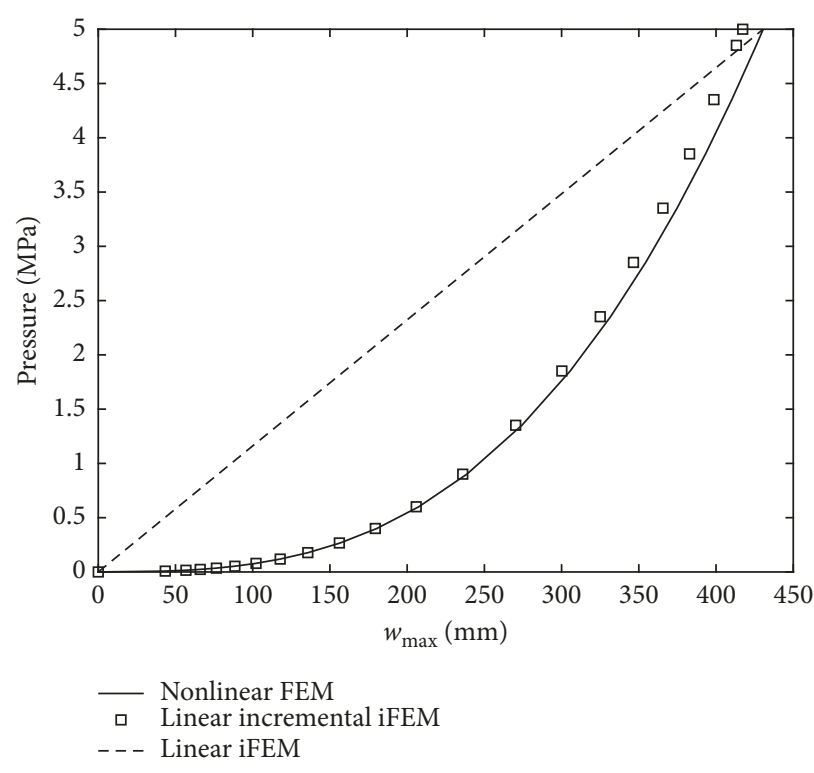

FIGURE 8: Clamped square plate. Load-deflection curves corresponding to the nonlinear direct FEM and linear iFEM analyses. The maximum deflection $\left(w_{\max }\right)$ is experienced at the plate center.

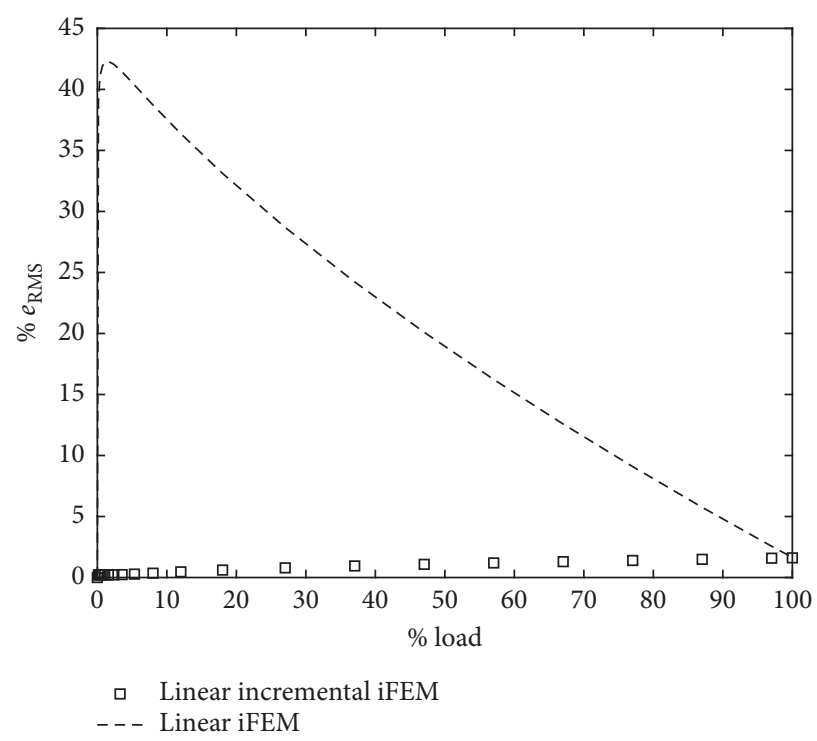

FIgURE 9: Clamped square plate. RMS percent error of the maximum deflection corresponding to the linear iFEM and linear incremental iFEM as a function of the load range.

good accuracy. This serves as further proof of the robustness of the linear incremental iFEM procedure.

\section{Conclusions}

The inverse problem of reconstructing the displacement field from the discrete strain-sensor data for plate and shell structures undergoing large deformations is addressed using the inverse Finite Element Method. The formulation is based on an incremental procedure, where at each load step, iFEM is applied using the incremental strains to obtain the 


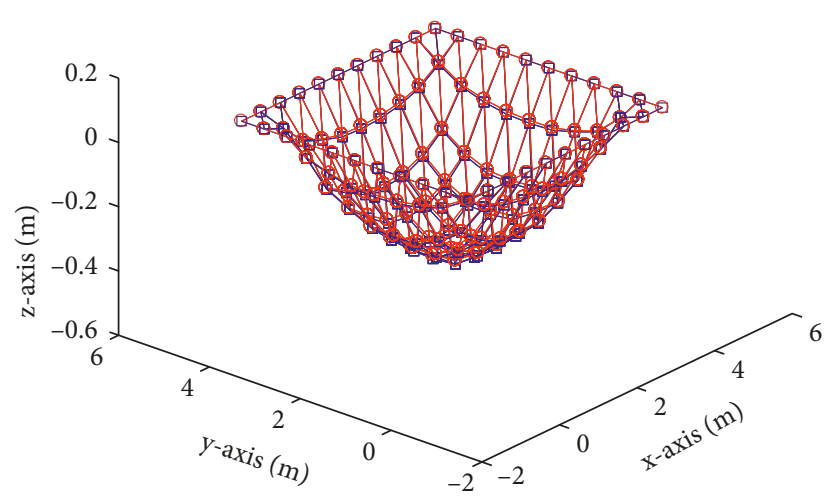

FIGURE 10: Clamped square plate. Deformed shape at the maximum load corresponding to the nonlinear direct FEM (blue) and linear incremental iFEM (red) solutions.

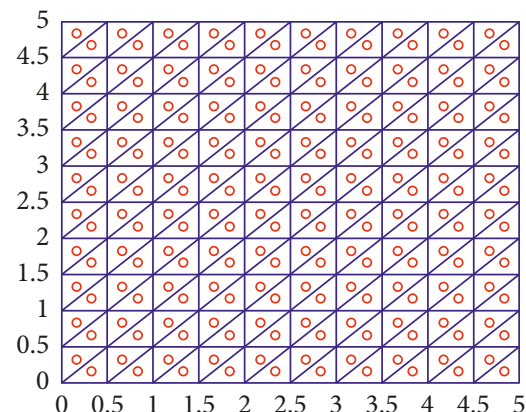

(a)

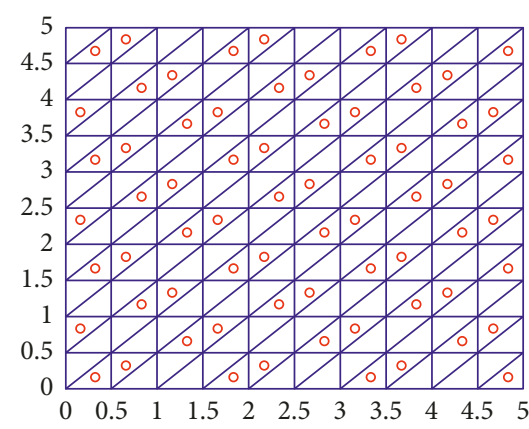

(c)

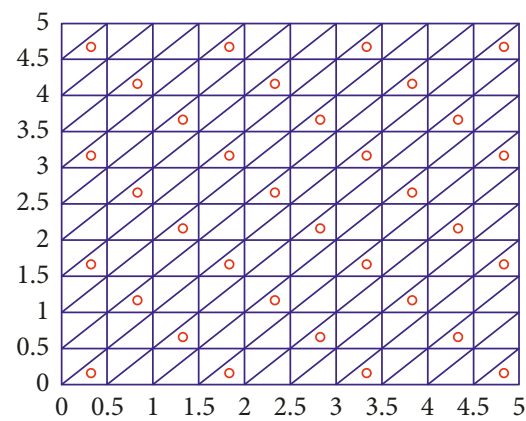

(e)

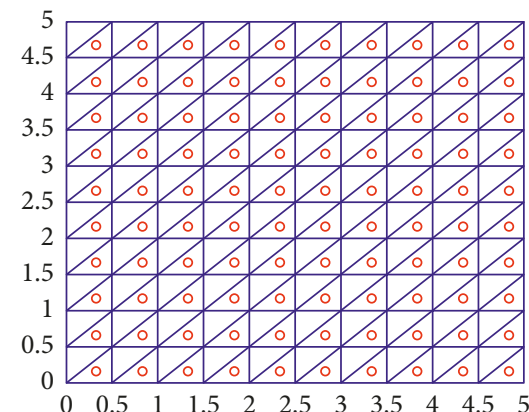

(b)

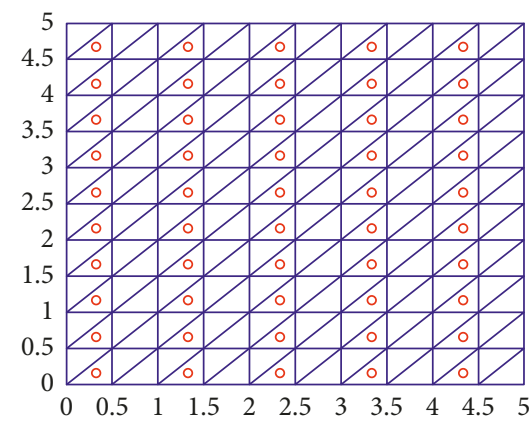

(d)

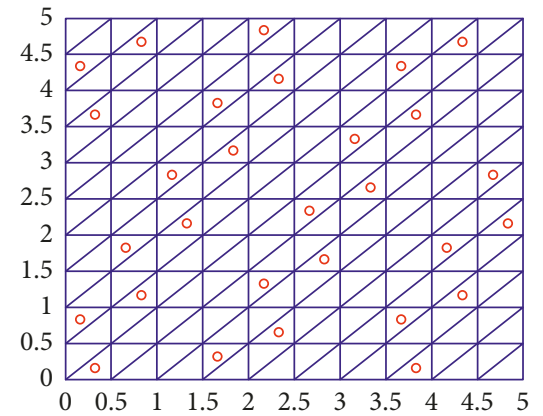

(f)

FiguRE 11: Six sensor arrangements used in the parametric study: (a) 200 strain gauges $(\beta=8)$, (b) 100 strain gauges $(\beta=4)$, (c) 67 strain gauges $(\beta=2.68)$, (d) 50 strain gauges $(\beta=2)$, (e) 34 strain gauges $(\beta=1.36)$, and (f) 29 strain gauges $(\beta=1.16)$. 


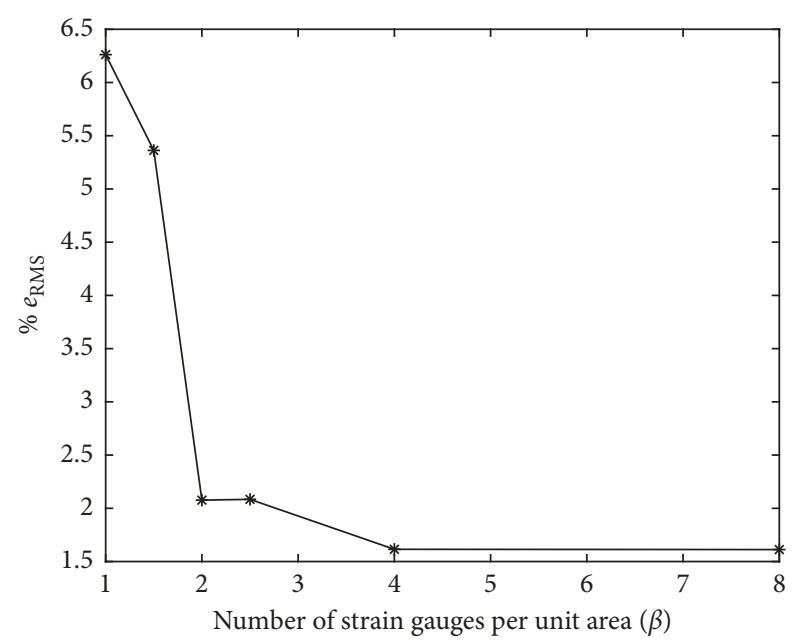

FIGURE 12: RMS percent error using linear incremental iFEM analysis for different sensor arrangements.

incremental displacements that are subsequently used to update the current deformed geometry.

To demonstrate the accuracy of the incremental iFEM approach, two numerical case studies have been carried out: (1) a cantilevered wing-shaped plate and (2) a clamped square plate subjected to transverse loading. To provide the incremental strain measurements as input data and displacements as reference data for the iFEM analysis, geometrically nonlinear direct FEM models were used. The iFEM accuracy was assessed by calculating the RMS of the nodal transverse deflection error. The incremental iFEM approach was demonstrated to be highly accurate and leads to precise load-displacement curves. Furthermore, the incremental iFEM procedure was demonstrated to be robust and accurate even when fairly sparse strain-sensor data is available, with many inverse elements without any strain data, and with only a single "measured" strain component used in the elements that had strain data.

The results of this paper clearly indicate that the incremental iFEM approach can be readily implemented as a viable real-time structural health monitoring tool for aerospace structures undergoing nonlinear deformations. Our future research will focus on the iFEM applications to geometrically nonlinear dynamic problems.

\section{Data Availability}

The FEM data used to support the findings of this study are available from the corresponding author upon request.

\section{Conflicts of Interest}

The authors declare that they have no conflicts of interest.

\section{References}

[1] M. Gherlone, P. Cerracchio, and M. Mattone, "Shape sensing methods: review and experimental comparison on a wing-shaped plate," Progress in Aerospace Sciences, vol. 99, pp. 14-26, 2018.
[2] W. Akl, S. Poh, and A. Baz, "Wireless and distributed sensing of the shape of morphing structures," Sensors and Actuators A: Physical, vol. 140, no. 1, pp. 94-102, 2007.

[3] W. L. Ko, W. L. Richards, and W. L. Fleischer, Applications of the Ko Displacement Theory to the Deformed Shape Predictions of the Doubly-Tapered Ikhana Wing, NASA/TP-2009-21465, NASA, Washington, DC, USA, 2009.

[4] M. A. Davis, A. D. Kersey, J. Sirkis, and E. J. Friebele, "Shape and vibration mode sensing using a fiber optic bragg grating array," Smart Materials and Structures, vol. 5, no. 6, pp. 759-765, 1996.

[5] H. J. Bang, S. W. Ko, M. S. Jang, and K. I. Kim, "Shape estimation and health monitoring of wind turbine tower using a FBG sensor array," in Proceedings of IEEE Instrumentation and Measurement Technology Conference, pp. 496-500, Graz, Austria, May 2012.

[6] L. H. Kang, D. K. Kim, and J. H. Han, "Estimation of dynamic structural displacements using fiber Bragg grating strain sensors," Journal of Sound and Vibration, vol. 305, no. 3, pp. 534-542, 2007.

[7] S. Rapp, L. H. Kang, J. H. Han, U. C. Mueller, and H. Baier, "Displacement field estimation for a two-dimensional structure using fiber Bragg grating sensors," Smart Materials and Structures, vol. 18, no. 2, article 025006, 2009.

[8] R. T. Jones, D. G. Bellemore, T. A. Berkoff et al., "Determination of cantilever plate shapes using wavelength division multiplexed fiber Bragg grating sensors and a leastsquares strain-fitting algorithm," Smart Materials and Structures, vol. 7, no. 2, pp. 178-188, 1988.

[9] Z. Mao and M. Todd, "Comparison of shape reconstruction strategies in a complex flexible structure," in Proceedings of the SPIE 6932, Sensors and Smart Structures Technologies for Civil, Mechanical, and Aerospace Systems, San Diego, CA, USA, March 2008.

[10] A. Tessler and J. L. Spangler, A Variational Principle for Reconstruction of Elastic Deformation of Shear Deformable Plates and Shells, NASA/TM-2003-212445, NASA, Washington, DC, USA, 2003.

[11] A. Tessler and J. L. Spangler, "A least-squares variational method for full-field reconstruction of elastic deformations in shear-deformable plates and shell," Computer Methods in Applied Mechanics and Engineering, vol. 194, no. 2-5, pp. 327-339, 2005.

[12] A. Tessler and J. L. Spangler, "Inverse FEM for full-field reconstruction of elastic deformations in shear deformable plates and shells," in Proceedings of the 2nd European Workshop on Structural Health Monitoring, Munich, Germany, July 2004.

[13] M. Gherlone, P. Cerracchio, M. Mattone, M. Di Sciuva, and A. Tessler, "Shape sensing of 3D frame structures using an inverse finite element method," International Journal of Solids and Structures, vol. 49, no. 22, pp. 3100-3112, 2012.

[14] A. Tessler, J. Spangler, M. Gherlone, M. Mattone, and M. Di Sciuva, "Deformed shape and stress reconstruction in plate and shell structures undergoing large displacements: application of inverse finite element method using fiber-Bragggrating strains," in Proceedings of 10th World Congress on Computational Mechanics, Sao Paulo, Brazil, 2012.

[15] Dassault Systemes Simulia Corp., Abaqus Analysis User's Guide (Version 6.13), Dassault Systemes Simulia Corp., Providence, RI, USA, 2013. 


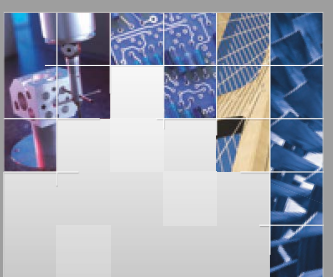

\section{Enfincering}
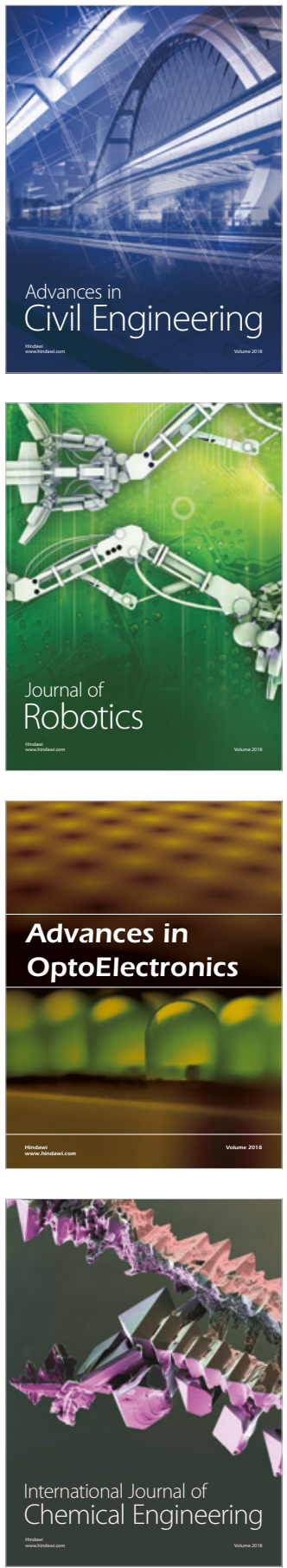

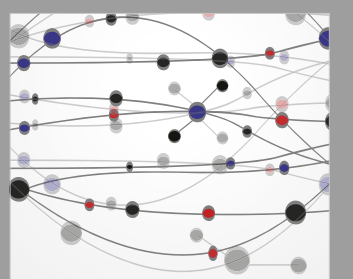

\section{Rotating \\ Machinery}

The Scientific World Journal

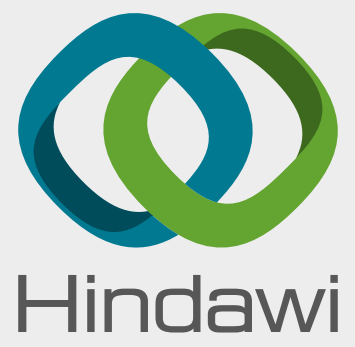

Submit your manuscripts at

www.hindawi.com
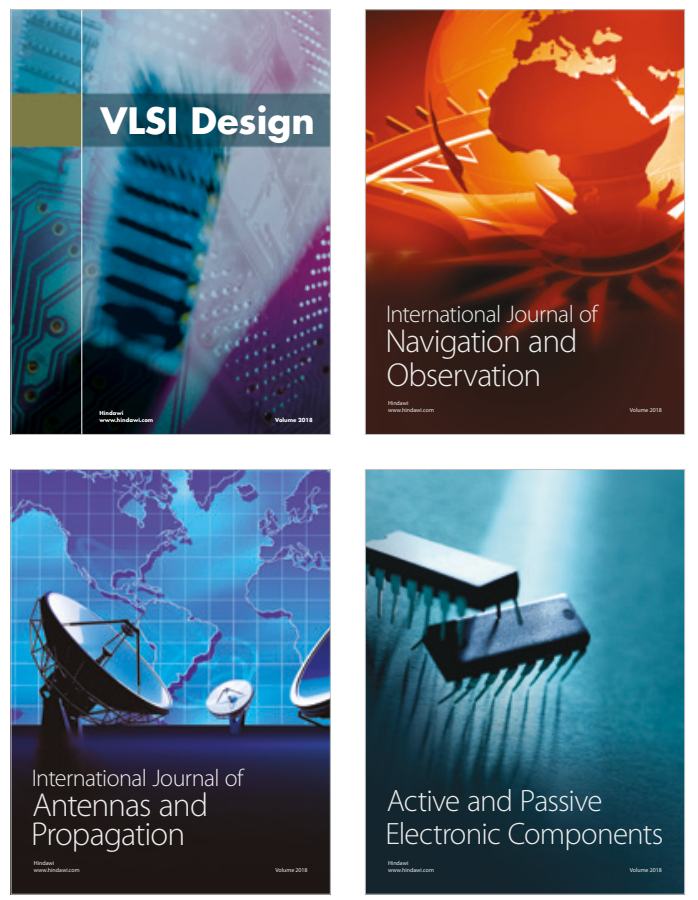
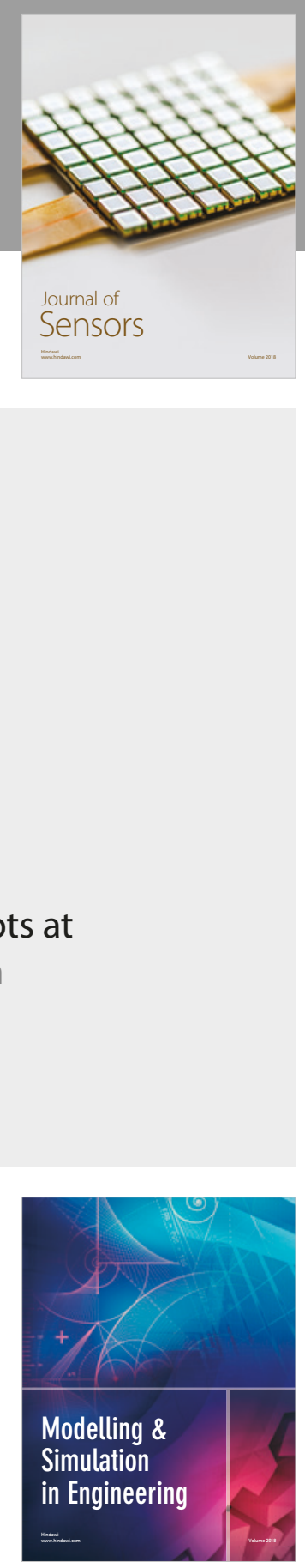

\section{Advances \\ Multimedia}
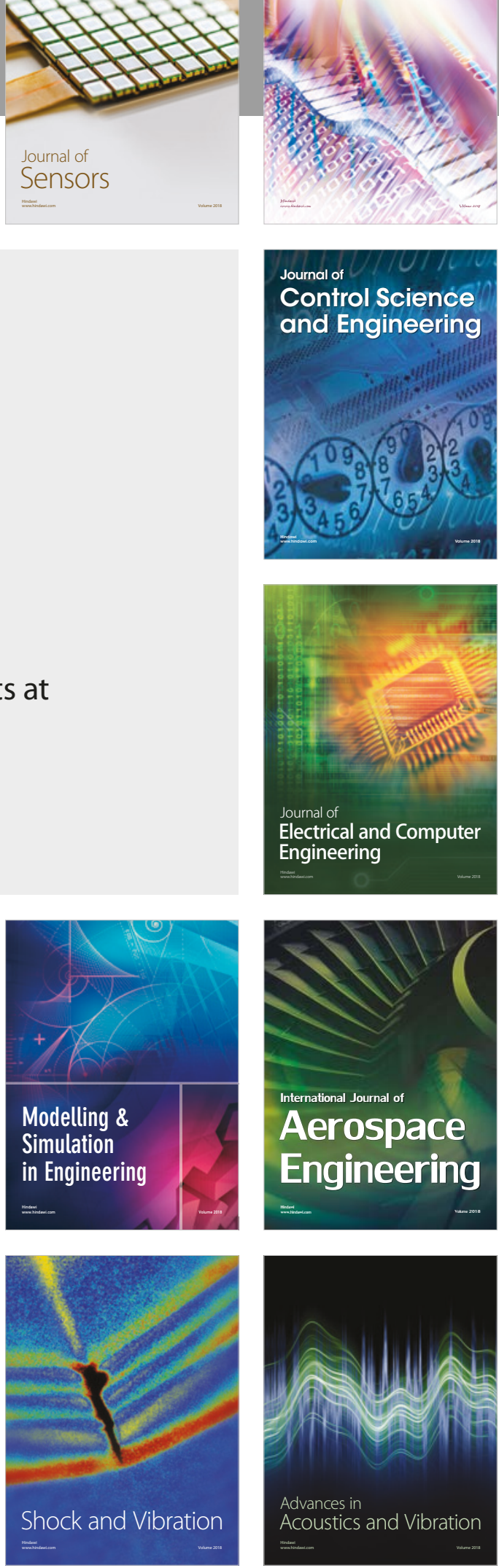